\title{
First principles calculation of inhomogeneous broadening in solid-state cw-EPR spectroscopy.
}

\author{
Hossam Elgabarty, ${ }^{\dagger} \ddagger$ Milian Wolf, ${ }^{\dagger}$ Adrian Glaubitz, ${ }^{\dagger}$ Dariush Hinderberger, ${ }^{\mathrm{gl}}$ and \\ Daniel Sebastiani*,§
}

Dahlem Center for Complex Quantum Systems (DCCQS), Physics Department, Freie Universität Berlin, Arnimallee 14, 14195 Berlin, Germany., Johannes Gutenberg University Mainz, Institute of Physical Chemistry and Center for Computational Sciences, Staudinger Weg 7 D-55128 Mainz,

Germany., The Max Planck Institute for Polymer Research (MPIP), Ackermannweg 10, 55128

Mainz, Germany, and Institute of Chemistry, Martin Luther University Halle-Wittenberg (MLU), Von-Danckelmann-Platz 4, 06120 Halle (Saale)

E-mail: daniel.sebastiani@chemie.uni-halle.de

\section{Abstract}

We present a scheme for the first-principles calculation of EPR lineshapes for continuous-waveEPR spectroscopy (cw-EPR) of spin centers in complex chemical environments. We specifically focus on poorly-characterized systems, e.g. powders and frozen glasses with variable microsolvation structures. Our approach is based on ab-initio molecular dynamics simulations and ab-initio calculations of the ensemble of g- and A-tensors along the trajectory.

The method incorporates temperature effects as well as the full anharmonicity of the intra- and intermolecular degrees of freedom of the system. We apply this scheme to compute the lineshape of a prototypical spin probe, the nitrosodisulfonate dianionic radical (Fremy's salt), dissolved in a 50:50 mixture of water and methanol. We are able to determine the specific effect of variations of local solvent composition and microsolvation structure on the $\mathrm{cw}-\mathrm{EPR}$ lineshape.

Our molecular dynamics reveal a highly anisotropic solvation structure with distinct spa-

\footnotetext{
*To whom correspondence should be addressed

${ }^{\dagger} \mathrm{DCCQS}$

$\ddagger \mathrm{JGU}$

IIMPIP

$\S_{\mathrm{MLU}}$
}

tial preferences for water and methanol around the Fremy's salt that can be traced back to a combination of steric and polar influences. The overall solvation structure and conformational preferences of the Fremy's salt as found in our MD simulations agree very well with the results obtained from EPR and orientation-selective ENDOR spectroscopy performed on the frozen glass. The simulated EPR lineshapes show good agreement with the experimental spectra. When combined with our MD results, they provide deep insights into the origin of local variations in the $g$ and A-tensors between different spin centers at variable local solvation environments, and how this can be traced back to a combination of intramolecular and intermolecular interactions.

\section{Introduction}

The determination of the detailed microscopic structure and dynamics of complex aqueous solutions is still a challenge for modern physics and chemistry. Unlike the situation in crystalline systems, where scattering experiments can provide very accurate atomic coordinates, aqueous systems lack the required long-range order, which limits the applicability of these scattering techniques. Electron paramagnetic resonance (EPR) 
is able to probe the local structure around an open shell system without the need for long-range order, and at the same time with high sensitivity to the local chemical environment. The two observables from an EPR experiment - the A-tensor and the g-tensor - are well known to be highly sensitive to local environmental changes like solvent polarity and fluctuations in the hydrogen bonding network. $^{1,2}$ However, the interpretation of the rich and detailed information presented by EPR spectroscopy has always been challenging and EPR spectroscopists are always looking for more elaborate tools to interpret their spectra, which basically comes down to assignment and interpretation of spin Hamiltonian parameters and of spectral lineshapes. The effective spin Hamiltonian represents the common grounds where results from theory and experiment converge. In this regard, the introduction of DFT was a turning point for the calculations of the spin Hamiltonian parameters, for it has been shown to provide remarkably accurate values at reasonable computational costs. ${ }^{3-10}$

In a previous publication we have characterized the complex solvation environment and hydrogen bonding network around the Fremy's salt (FS, see Figure 1) in frozen water-methanol binary solvent by means of EPR/ENDOR spectroscopy and forcefield molecular dynamics simulations. ${ }^{11}$ Like other nitroxide free radicals, FS is a chemically stable free radical, and its spectroscopic signatures are strongly sensitive to the chemical surroundings, ${ }^{12}$ which makes it a suitable probe to study ionic solvation by EPR spectroscopy. In that work we had used classical MD simulations to help explain the experimental results. In particular, the solvation structure revealed by MD simulations was found to qualitatively agree with evidence coming from orientation-selective ENDOR.

Here, we present a more quantitative description of the solvation environment by means of $a b$ initio MD simulations combined with first principles calculations of the spin Hamiltonian parameters. We also focus on how local disorder in the solvation structure in such a heterogenous frozen glass gives rise to the overall inhomogeneously broadened spectral envelope observed in continuous wave EPR spectroscopy (cw-EPR). To this end, we examine and correlate the variation in spin Hamiltonian parameters with both intermolecular (solvation) parameters as well as intramolecular ones (the geometry of the FS itself).
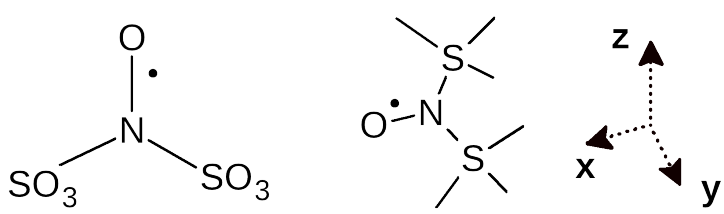

Figure 1: The Fremy's salt. The axis system we use is depicted on the right side.

Solid-state spectra are generally inhomogeneously broadened, which in the context of EPR refers to the situation where the ensemble of spins in the sample has a distribution of resonance frequencies and widths, with the individual widths being much less than the total width of the frequency distribution i.e. the distribution of the effective field at different spins is broader than the natural linewidth. This happens when the surrounding lattice is much slower than the resonance phenomenon, as exactly is in our case. ${ }^{13,14}$ In this situation the overall spectral line consists of many lines merged under one envelope. Although inhomogeneities of the static field and unresolved fine/hyperfine structure do play role in the line broadening, however, the two major nonrelaxational sources of broadening in this case are $g$ and A-tensor anisotropies. ${ }^{15,16}$ At each spin center, the values of the $g$ and A-tensors are a complicated function of both intramolecular as well as intermolecular (solvation) factors, and it is usually the task of the spectroscopist to infer useful insight about the (possibly heterogenous) solvation structure and the geometry of the free radical from the average lineshape. Furthermore, in the case of a powder spectrum, the lineshape is an average over all the possible orientations of the tensors relative to the applied magnetic field.

A number of studies that combine $a b$ initio MD simulations and theoretical EPR spectroscopy have come out. ${ }^{10,17,18}$ These studies indeed demonstrate the strength and utility of this approach. The previous studies were either focused on aqueous solutions or crystalline solids. Thus they either dealt with a system in the "motionally narrowed" limit or a system embedded a regular periodic lattice. To the best of our knowledge this is the first ab initio MD/EPR study of 
a heterogenous system in a frozen glassy state. Although methanol-water is probably one of the most simple binary solvents, the current general consensus is that it is far from being homogeneous. Experimental evidence and theoretical modeling suggest that these solutions are microheterogenous, separating into methanol-rich regions and water-rich regions. ${ }^{19}$ When frozen, the mixture forms a glass which can be thought of as containing fixed randomly oriented spin centers lacking any spatial or orientational correlation. In such a heterogenous frozen glass, not only the relative orientation or distance between solvent molecules and the FS may vary from site to site, but also the local numbers of water or methanol molecules also vary. Such poorly characterized systems present special challenges in EPR spectroscopy. ${ }^{20}$ In order to capture such heterogeneity, and its effects of the inhomogeneously broadened EPR lineshape we have designed a simulation scheme as depicted in Figure 2. 30 ab initio MD trajectories were started at regular time points from a classical MD trajectory. Each ab initio MD trajectory was run at a constant temperature of $300 \mathrm{~K}$ for $3.5 \mathrm{ps}$, followed by annealing down to $200 \mathrm{~K}$ in $3 \mathrm{ps}$, and a final rapid annealing down to $20 \mathrm{~K}$ in $1 \mathrm{ps}$. The final configuration from each of the annealed trajectories was used to compute spin Hamiltonian parameters (g and A-tensors, chemical shifts, quadrupolar couplings), and then finally used to construct an EPR lineshape. EPR lineshapes were computed via exact diagonalization of the spin Hamiltonian as described in the Computational Details.

\section{Computational details}

MD Simulations. From a 10 ns classical MD trajectory, ${ }^{11} 30$ snapshots were extracted by uniform sampling. From each of them, a sub-cell containing the Fremy's salt and the first two solvation shells was extracted and used for subsequent $a b$ initio MD simulations (Figure 2). In the large simulation cell the methanol molar ratio $(0.308)$ corresponds to a 50:50 volume ratio at room temperature. In the 30 sub-cells the methanol molar ratio was found to vary between 0.23 to 0.38 , with an average of 0.309 (Figure 1).

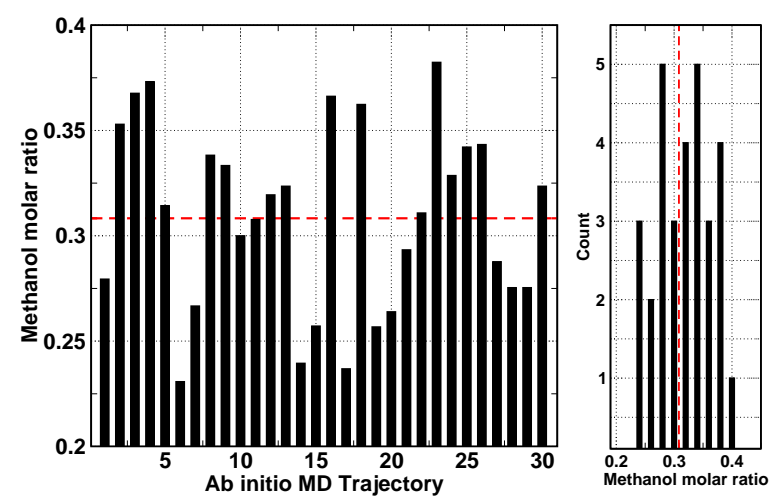

Graph 1: Methanol molar ratio in each of the thirty $\mathrm{ab}$ initio MD trajectories. The dotted line marks the experimental (and classical MD) molar ratio.

$\mathrm{Ab}$ initio MD simulations were performed with $\mathrm{CP} 2 \mathrm{~K}^{21}$ using the Gaussian and plane waves (GPW) method with a DZ-MOLOPT-SR basis set $^{22}$ and a planewave density cutoff of $320 \mathrm{Ry}$. The PBE functional ${ }^{23}$ was used together with the Grimme D2 dispersion correction. ${ }^{24}$ After an initial isothermal relaxation period of $3.5 \mathrm{ps}(300 \mathrm{~K}$, CSVR thermostat, ${ }^{25} \tau=500 \mathrm{fs}$ ), the system was annealed in two steps: A slow annealing down to $220 \mathrm{~K}$ during 3 ps, followed by a faster annealing down to $20 \mathrm{~K}$ in $1 \mathrm{ps}$. Thus each of the $30 \mathrm{ab}$ initio MD trajectories had a total length of 7.5 ps.

EPR Simulations. For calculations of EPR spectroscopic parameters, only the Fremy's salt and the first solvation shell were considered. The first solvation shell here is defined as any solvent molecule with at least one atom within three Angstroms from any FS atom. This choice was based on an explicit calculation of the target quantities at various cluster sizes up to full periodic boundary conditions. Three different setups were used for the calculation of EPR g-tensors (Table 1), all of them using the IGLO-III basis set. ${ }^{26}$ The setup employing $\mathrm{CP} 2 \mathrm{~K}$ used the all-electron GAPW method, ${ }^{27,28}$ and the Wavelet Poisson solver was used to decouple from periodic images. ${ }^{29}$ A-tensors were computed in Orca ${ }^{9,30}$ using the PBE0 functional and the EPR-III basis set. ${ }^{31}$ The validity of these setups, particularly regarding the choice of the exchange correlation functional and the basis set, has been validated in different previous studies. ${ }^{7-10}$ Additional core STO-basis functions were added to the Nitrogen atom of the Fremy's salt (CORE_DZ_ADF basis 

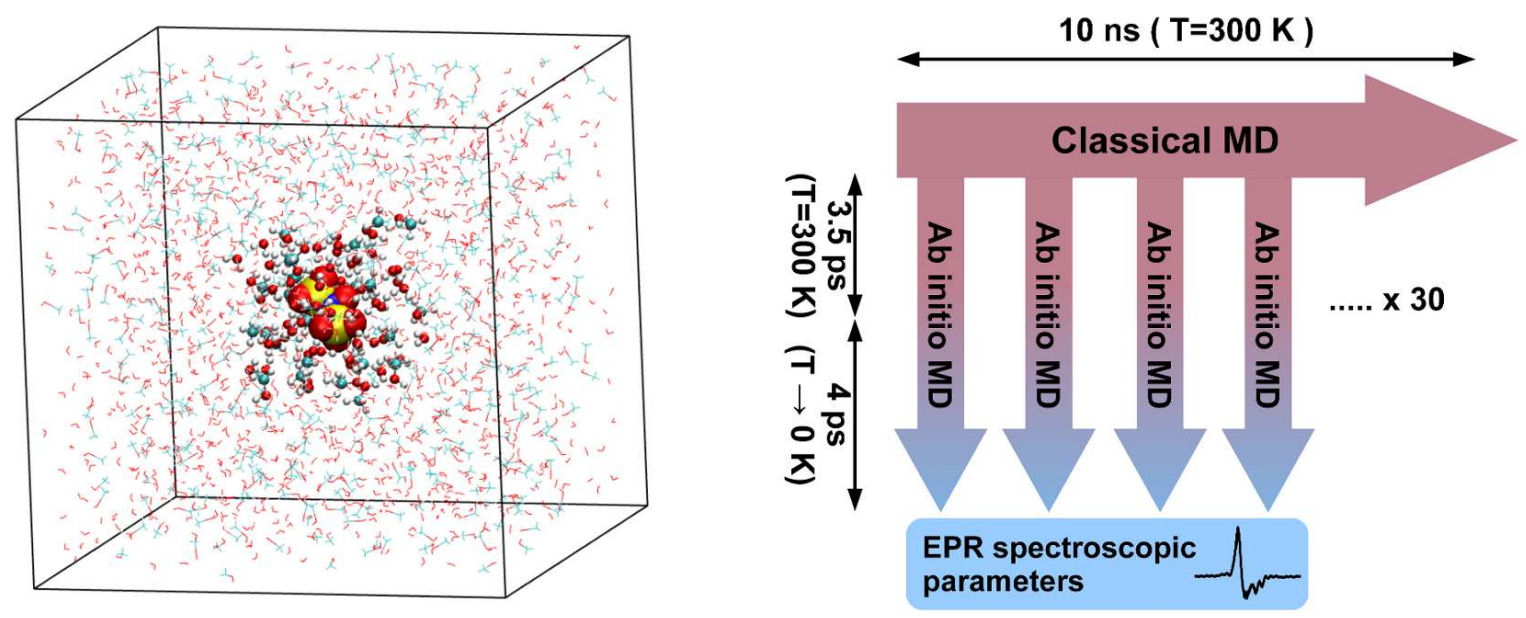

Figure 2: Left: A snaphot of Fremy's salt solvated in methanol and water taken from a classical (force field) trajectory. The central part depicted in spheres represent the sub-system that is extracted for ab initio MD. Right: Simulation scheme.

set in Orca). The inclusion of theses core STO functions was found to have a relatively weak ($3 \mathrm{MHz}$ ) but statistically significant influence on the value of the isotopic part of the hyperfine coupling. Statistical analyses were done using $\mathrm{R},{ }^{32-34}$ all the results reported as statistically significant have a p-values less than 0.001 .

EPR spectral line shapes were simulated using a frequency domain approach via exact diagonalization of the spin Hamiltonian using our own $\mathrm{C}++$ code. The Spin Hamiltoninian included the following terms:

$$
\hat{\mathscr{H}}=\mu_{B} B_{0} g S+\sum_{i} S A_{i} I_{i}-\mu_{N} \sum_{i} g_{N} I_{i} B_{0}+I Q I
$$

Where the terms from left to right are the electron Zeeman term, the hyperfine coupling, the nuclear Zeeman terms, and the quadrupolar coupling (only for nitrogen). Only the five most strongly coupled solvent hydrogens were included in the simulation and chemical shift anisotropy was ignored. Adaptive segmentation of the field values was implemented, ${ }^{36}$ and powder averaging was performed on a Lebdev 101 hemispherical grid $^{37,38}$ (MPI-parallelized). We do not include an explicit relaxation term in the spin Hamiltonian, instead we account for the damping of the spin coherences (transverse relaxation) phenomenologically by convoluting the frequency domain spectrum with a decaying exponential. ${ }^{39}$

\section{Results and Discussion}

\section{Solvation Dynamics of the Fremy's Salt}

Table 2 gives averaged internal coordinates of the solvated Fremy's salt at $300 \mathrm{~K}$ and $20 \mathrm{~K}$. The most interesting point here is possibly the improper torsional angle between the FS nitrogen, the two sulfurs, and the nitroxy oxygen, which we find to be slightly off-planar. This agrees with the results previously reported by Hinderberger et al based on rotational diffusion tensors from the simulation of cw-EPR spectra. ${ }^{40}$

Table 2: MD-averaged internal coordinates of the Fremy's salt. The values for the improper angle are unsigned averages. The averages at $300 \mathrm{~K}$ were obtained by averaging over all the $300 \mathrm{~K}$ trajectories, while the annealed averages were obtained only from the configurations that were used to compute the spectroscopic parameters (i.e. last configuration from each trajectory).

\begin{tabular}{l|c|c} 
Coordinate & MD-Average $(300 \mathrm{~K})$ & Annealed \\
\hline $\mathrm{N}-\mathrm{O}$ & $1.275 \pm 0.024 \AA$ & $1.272 \AA$ \\
$\mathrm{N}-\mathrm{S}$ & $1.858 \pm 0.063 \AA$ & $1.843 \AA$ \\
$\mathrm{S}-\mathrm{N}-\mathrm{S}$ & $121.4 \pm 4.1^{\circ}$ & $121.3^{\circ}$ \\
$\mathrm{N}-\mathrm{O}_{\mathrm{N}}-\mathrm{S}-\mathrm{S}$ & $12.3 \pm 7.3^{\circ}$ & $13.2^{\circ}$
\end{tabular}

Regarding solvent distribution around the 
Table 1: The three setups used to compute the g-tensor

\begin{tabular}{l|c|c} 
& XC-functional & Gauge origin \\
\hline Setup 1: Gaussian 09 & PBE0 & GIAO \\
Setup 2: Orca 2.99,30 & PBE0 & Center of electronic charge \\
Setup 3: CP2K $(G A P W)^{28}$ & PBE & CSGT
\end{tabular}

Fremy's salt, Figure 3 shows the radial distribution function of the polar hydrogens around the FS nitroxy oxygen at $300 \mathrm{k}$ and at $20 \mathrm{~K}$. At $300 \mathrm{~K}$ sulfonyl oxygens have a tighter and stronger solvation shell compared to the nitroxy oxygen (coordination numbers 1.40 and 0.73 respectively). At $20 \mathrm{~K}$, the solvation structure gets more ordered, with the coordination at the sulfonyl oxygens remaining exactly the same. In contrast, the coordination number at the nitroxy oxygen increases to 1 . Close inspection reveals that the first solvation shell in the latter case is in fact composed of two gaussians, centered at $1.83 \AA$ and $2.05 \AA$ (See inlet of Figure 3). This matches our previous findings from cw-EPR spectra for this system, where two different $g_{x x}$ values were required to fit the low-field end of the experimental spectrum to an empirical spin Hamiltonian.

Figure 4 shows the spatial distribution of methyl hydrogens around the FS, upto a cutoff distance of $3.5 \AA$. The RDF and the SDF combined give a concrete picture which closely matches previous theoretical and experimental EPR and orientationselective ENDOR findings. ${ }^{11}$ Quantitatively, our forcefield MD gives a tighter solvation shell compared to PBE-D2.

To summarize, the anisotropy of solvent configuration around the salt can be attributed to a combination of polar and steric factors, whence polar factors manifest themselves in the different strengths of the sulfonyl oxygens and the nitroso oxygen as hydrogen bond acceptors, while the steric factor is clearly seen in the hindrance caused by the sulfonyl groups and their solvation shell, which interferes with solvent accessibility to the nitroxy oxygen. Figure 5 depicts a typical solvation pattern around the FS.
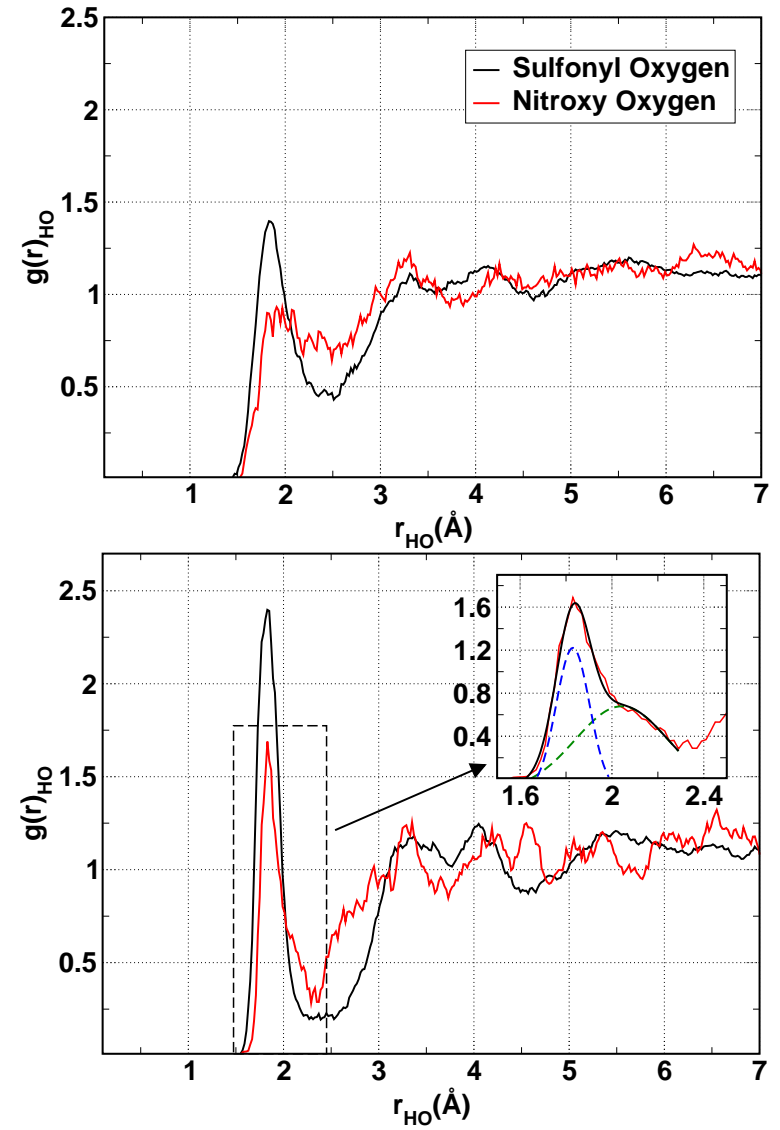

Figure 3: RDF of the polar hydrogens around the FS nitroxy oxygens. Top: $300 \mathrm{~K}$, bottom: $20 \mathrm{~K}$.
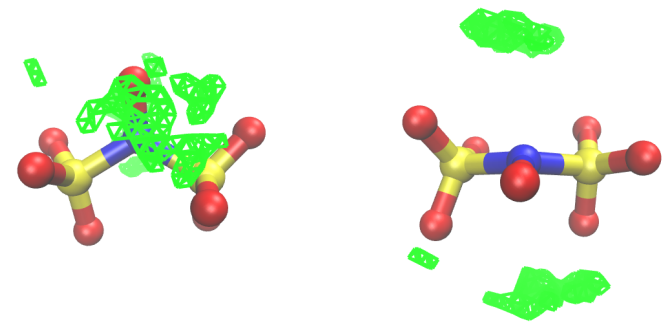

Figure 4: Spatial distribution of the non-polar (methyl) hydrogens around the Fremy's salt 


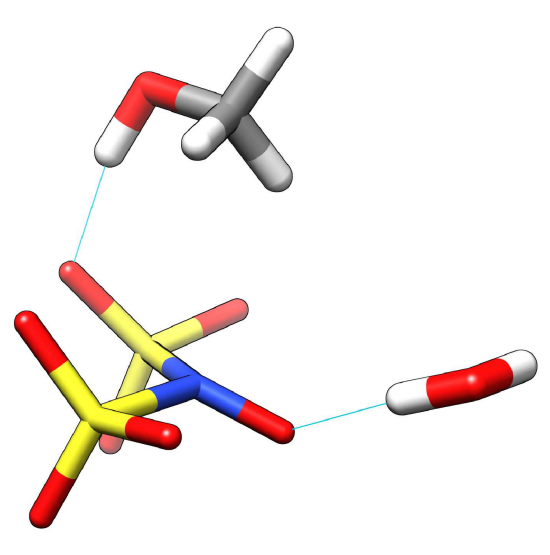

Figure 5: Typical solvation pattern of the Fremy's salt.

\section{EPR spectral parameters}

\section{Hyperfine coupling to nitrogen}

At the W-band, the hyperfine coupling to the FS nitrogen atom is the second strongest term in the spin Hamiltonian after the electron Zeeman term. Figure 6 shows annealed the MD-averaged principal values of the total nitrogen hyperfine coupling tensor, which shows excellent agreement with experiment.

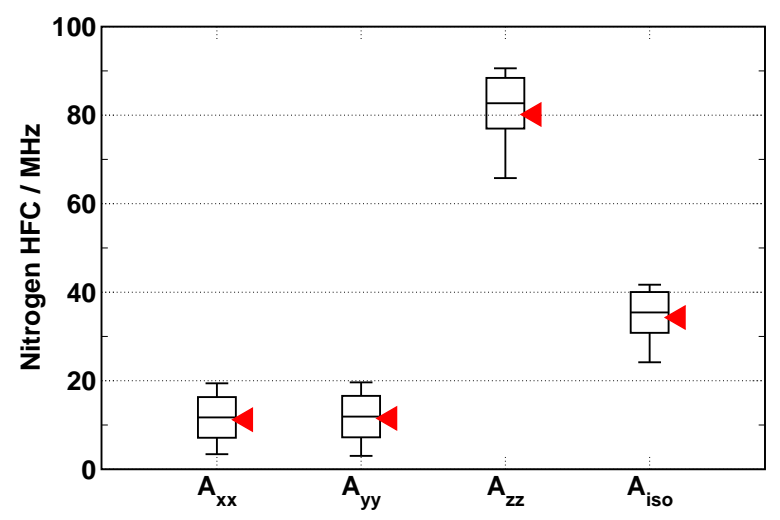

Figure 6: Averaged principal values of the computed total nitrogen hyperfine coupling tensor (Center bar of each rectangle), their standard deviation (upper/lower border of rectangle), and minimum/maximum values from the sampling (error bars). The red triangles mark the experimental values

It turns out that the value of $\mathrm{A}_{\text {iso }}$ is strongly correlated with the improper torsional angle of FS (Figure 7 left), which alone accounts for $75 \%$ of the variation in $\mathrm{A}_{\mathrm{iso}}$. We have also tested regression models relating $\mathrm{A}_{\text {iso }}$ to other FS geometric parameters. In addition, we included two parameters related to the solvation of the nitroxy group: the shortest hydrogen bond length, and the shortest distance to a methyl hydrogen. We found that in addition to the improper torsion, the inclusion of the nitroxy $\mathrm{N}-\mathrm{O}$ distance and the hydrogen bond length to NO gives a model with an adjusted $R^{2}$ of 0.88 (supplementary information). Thus we find that almost $90 \%$ of the variation in $A_{i s o}$ is related to three simple geometric parameters: The cosine of the FS improper torsion, the $\mathrm{N}-\mathrm{O}$ bond length, and the hydrogen bond length to NO.

We now turn to the three principal components of the anisotropic HFC tensor. They only show a significant (but weak) correlation with the NO. . $\cdot$ H-bond length $\left(R^{2}=0.55-0.6\right)$. In our opinion, the more interesting quantity for this system is the rhombicity of the A-tensor $\left(\delta \mathrm{A}=\mathrm{A}_{X}-\mathrm{A}_{Y}\right)$, which already shows a stronger correlation with the NO $\cdots$ H length $\left(R^{2}=0.70\right.$, Figure 7 right $)$. Again, a stronger linear regression model $\left(R^{2}=\right.$ $0.85)$ employing three independent variables is given in the supplementary information.

To summarize, we found that different components of the hyperfine coupling tensor of the FS nitrogen atom carry different pieces of geometric information. $\mathrm{A}_{\mathrm{iso}}$ is highly sensitive to the $\mathrm{S}-\mathrm{N}-\mathrm{S}$ $\mathrm{O}$ imporoper torsion, and since this relation is surprisingly stable and almost insensitive to solvation effects, it can indeed be exploited to measure the degree of "planarity" of FS in different environments. On the other hand, the rhombicity of the A-tensor can be used as a probe for the hydrogenbonding to the nitroxy oxygen.

\section{Hyperfine coupling to solvent hydrogens}

Figure 8 depicts $A_{\text {iso }}$ and the Frobenius norm of $\mathrm{A}_{\text {aniso }}$ as related to the distance between the hydrogen and the center of the $\mathrm{N}-\mathrm{O}$ bond. The value of $A_{\text {iso }}$ is mostly very close to zero, reflecting the absence of any significant spin density at the solvent hydrogens. The only exception is the hydrogens that are strongly hydrogen bonded to the FS nitroxy, these acquire a slightly negative $A_{\text {iso }}$ due to spin polarization. Regarding the anisotropic part of the hyperfine coupling tensor, its Frobenius 

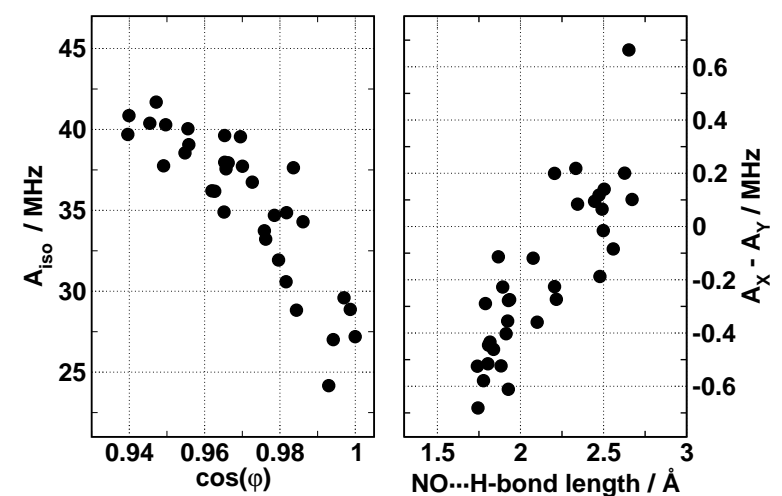

Figure 7: Scatterplots of nitrogen $A_{\text {iso }}$ against the FS improper torsion (Left) and of the nitrogen Atensor rhombicity $(\delta \mathrm{A})$ against the $\mathrm{NO} \cdots \mathrm{H}$-bond length (right).

norm shows the expected $r^{-3}$ distance dependence (Figure 8).

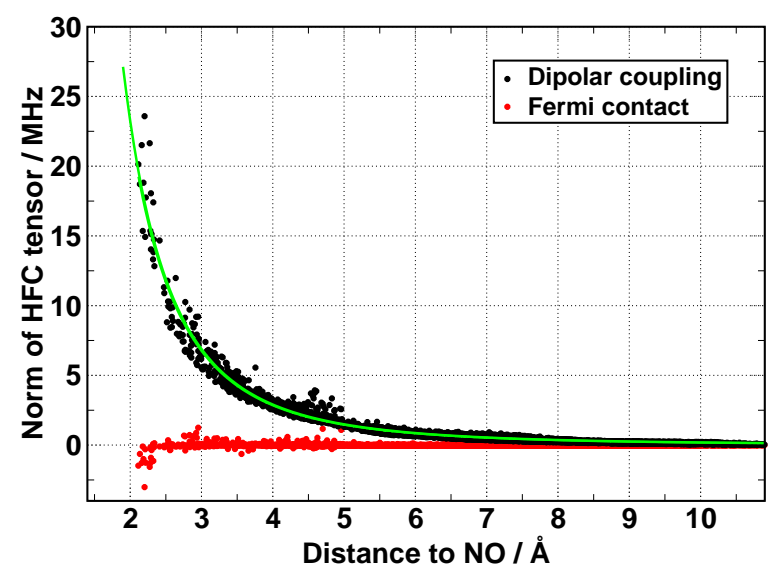

Figure 8: Hydrogen HFC as related to distance between the hydrogen and the center of the N-O bond. The green curve is a $r^{-3}$ decay starting from a linear fit of the first 20 points. Black: Frobenius norm of the anisotropic A-tensor. Red: $\mathrm{A}_{\text {iso }}$.

The point-dipolar hyperfine coupling to solvent hydrogens, as for instance observed in ENDOR spectroscopy, is related to the radial distribution of hydrogens around the unpaired electron density, weighted by $r^{-3}$. An interesting question in many situations is how far spatially can we see solvent protons in the ENDOR spectrum. Another related question is the spatial range that gives the strongest contribution to the ENDOR spectrum. From an experimental point of view, the FS is particularly suitable for addressing these questions because it lacks any hydrogens in its structure, thus any unresolved hyperfine couplings must come from the solvent. To tackle this question, we have weighted the radial distribution of the hydrogens by $r^{-3}$ (the best fitting curve in Figure 8), which is shown in Figure 9. This plot can be interpreted as the average norm of the hyperfine coupling tensor from hydrogens at a distance $r$, relative to the coupling coming from distant "matrix" hydrogens. From the plot it can be seen that the hyperfine coupling to polar hydrogens is most strong at a distance of $2.3 \AA$, following by a weaker but broader peak centered at $3.5 \AA$ A . Beyond $4.5 \AA$ the coupling rapidly decays to zero. Regarding the non-polar methyl hydrogens, the plot looks totally different, with a broad peak extending between 2.5 to $4 \AA$. One always has to keep in mind the anisotropic solvation picture previously discussed. Thus, for methyl protons the first peak originates from protons along the z-axis while the strong peak for polar protons reflects the hydrogen bonding to NO. If the plot is interpreted as a distribution density of hyperfine coupling, then first moment of the distribution is $3.8 \AA$ for polar protons and $4.2 \AA$ for non-polar ones.

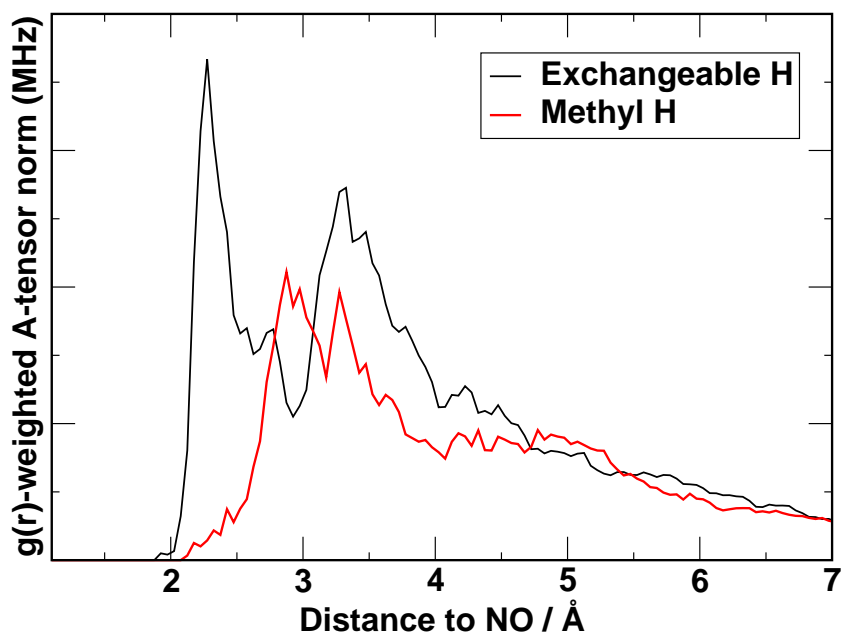

Figure 9: RDF of protons relative to the center of the $\mathrm{N}-\mathrm{O}$ bond weighted by $r^{-3}$.

\section{g-tensor}

Figure 10 shows the average g-tensor components obtained from the three setups ( Table 1) and Table 3 summarizes the errors compared to experiment. All the values are underestimated, especially the $\mathrm{g}_{\mathrm{xx}}$ component. However, errors of the order of $1000 \mathrm{ppm}$ are not uncommon in g-tensors 
computed from DFT, and the quality of these results is generally consistent with previous studies. ${ }^{9,10,30,41,42}$ Comparing the three setups, setup 1 gives the best match to experimental results, while Setup 2 gives a slightly better fit to $\mathrm{g}_{\text {iso }}$ and performs slightly better than setup 3 even though the former uses a GGA functional.

Solvent-induced shifts of the g-tensor are wellknown in EPR spectroscopy. It has been shown that the employed level of theory in this work is capable of accurately reproducing these effects using both explicit and implicit solvation models. ${ }^{43}$ Here, we also observe a correlation between the $\mathrm{g}_{\mathrm{xx}}$ and hydrogen bond length to NO (Supplementary information).

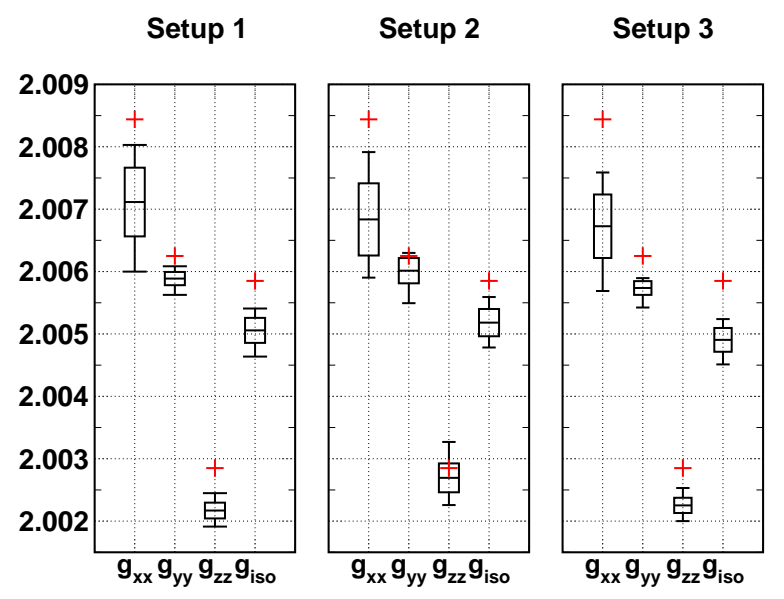

Figure 10: Left: Distribution of the principal gtensor components

Table 3: Error in g-shifts $\Delta \Delta g(\mathrm{ppm})$ relative to experimental values. ${ }^{11}$

\begin{tabular}{l|c|c|c} 
g-tensor component & Setup 1 & Setup 2 & Setup 3 \\
\hline $\mathrm{g}_{\mathrm{xx}}$ & -1326 & -1604 & -1714 \\
$\mathrm{~g}_{\mathrm{yy}}$ & -363 & -236 & -514 \\
$\mathrm{~g}_{\mathrm{zz}}$ & -681 & -156 & -598 \\
$\mathrm{~g}_{\text {iso }}$ & -793 & -667 & -945 \\
RMSD & 885 & 940 & 1089
\end{tabular}

\section{Ensemble-averaged EPR line shapes}

Figure 11 depicts the annealed MD-averaged cwEPR first-derivative lineshape. The lineshape which shows reasonable agreement with the experimental line, reproducing all the features except for the broad peak at the low-field end of the spectrum, corresponding to $\mathrm{g}_{\mathrm{xx}}$. The loss of detail in the low-field part of the spectrum is due to the inherent theoretical approximations which result in a somewhat larger numerical error for $\mathrm{g}_{\mathrm{xx}}$, but also partly attributed to the broad distribution of hydrogen bonding to NO. Figure 12 shows two MD-averaged lineshapes, one taken from snapshots that have a strong hydrogen bond to NO (hydrogen bond length maximum $1.8 \AA$ ), and another one from snapshots that are non-hydrogen bonded (closed hydrogen at least $2.4 \AA$ away). With this decomposition of the theoretical lineshape, we clearly see a small peak in the lineshape taken from the strongly solvated FS configurations. To summarize, the difficulty in obtaining a better match for the low-field end of the spectrum is mainly due to the accuracy limit of density functional theory regarding the calculation of the $\mathrm{g}_{\mathrm{xx}}$ component. This problem can in principle be fixed by increasing the level of theory for the g-tensor calculation, or applying a suitable aposteriori correction scheme. Nevertheless, the qualitative agreement of the lineshape in Figure 11 and Figure 12 is striking and illustrates the power of first-principles molecular dynamics simulations combined with theoretical spectroscopy.

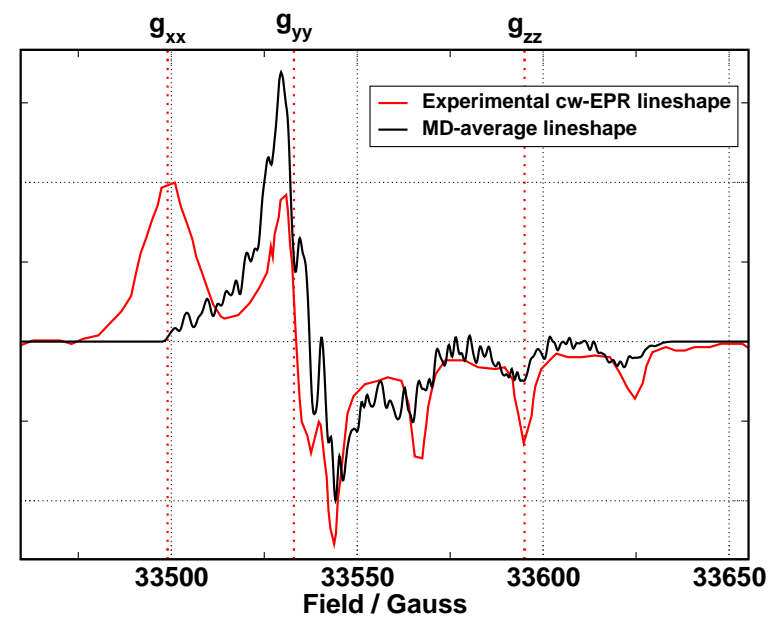

Figure 11: Annealed MD-averaged cw-EPR lineshape. The three dotted lines mark the experimental principal components of the g-tensor.

\section{Conclusions}

We have performed ab initio MD simulations of the Fremy's salt in a frozen glass of wa- 


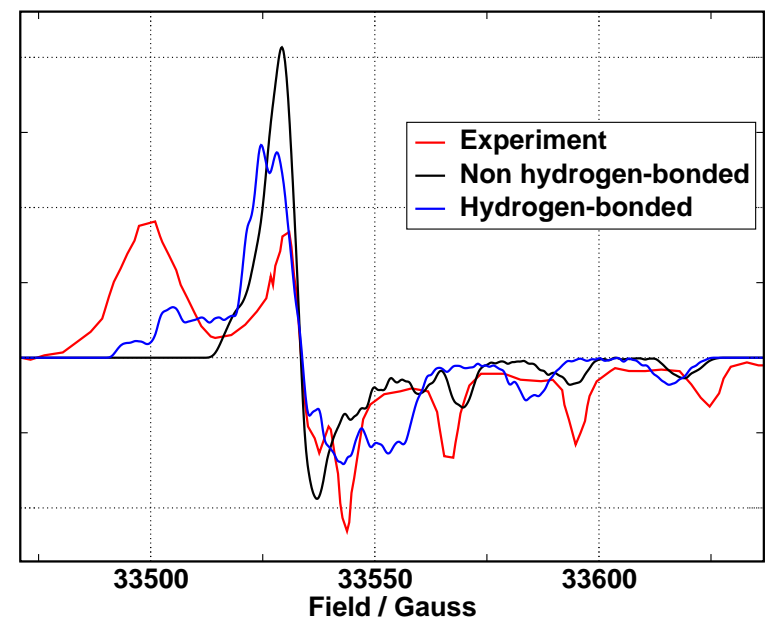

Figure 12: Decomposing the total annealed MDaveraged lineshapes into different lineshapes according to solvation of NO. Black lineshape: NO strongly hydrogen bonded, Red lineshape: NO not hydrogen bonded.

ter/methanol binary solvent. Such systems are often poorly characterized and present special challenges in EPR spectroscopy, in contrast to e.g. isotropic liquids or periodic solids. ${ }^{20}$ Specifically, we have explicitly sampled the local strain effects, so called "g-strain" and A-tensor anisotropy by first principles calculations of these quantities from 30 different annealed ab initio MD trajectories. The latter were derived from conventional force-field MD simulations, which yielded a specific distribution of local water:methanol molar ratios around the FS. We have also simulated the cwEPR lineshapes by explicit numerical diagonalization of the spin Hamiltonian.

Examination of the ab initio MD trajectories provides detailed insight into the structure of the solvation shell, which is found to be highly anisotropic with clear and distinct spatial preferences for water and for methanol. MD also provide details about hydrogen bonding networks, and the conformational preferences of the solvated salt, which all agree very well with evidence from EPR and ENDOR spectroscopy. ${ }^{11,40}$

We have also established a number of correlations between A and g-tensor components and inter/intra molecular geometric parameters. Generally, both tensor are most sensitive to the geometry of the FS itself, followed by solvation effects. Specifically, we found that $\mathrm{A}_{\text {iso }}$ is partic- ularly sensitive to the S-N-O-S improper torsion of the FS, and that this relation is insensitive to solvation effects, and thus can serve as an experimental measure of the planarity of the FS. We also found that the rhombicity of the A-tensor is related to the hydrogen bond between the nitroxy group and solvent. Based on the radial distribution functions of the solvent hydrogens, we were able to provide estimates of the strength of the hyperfine coupling of polar/nonpolar protons at different distances from the NO group, a quantity of direct relevance to ENDOR spectroscopy. Finally, decomposing the simulated lineshape shows good agreement with experiment, reproducing all the lineshape features except for the low-field peak corresponding to $\mathrm{g}_{\mathrm{xx}}$, which is attributed to the relatively large error in the corresponding computed quantity, and strong variation in this particular gtensor component with the (highly variable) hydrogen bond strength to the NO. Decomposing the total computed lineshape into two limiting situations: the strongly hydrogen bonded limit, and the non-solvated limit reveals the g-tensor strain corresponding to this variability in the NO solvation. It is worth noting here that the low-field peak of the experimental spectrum was not verywell reproduced also by the empirical spin Hamiltonian based on least-squares fitting, which was also attributed previously to g-tensor strain. Thus, even though this particular part of the experimental spectrum is not well-reproduced by our theoretical lineshape, we were still able to explicitly show how variation in the solvation structure is directly reflected in the lineshape.

Acknowledgement Financial support is gratefully acknowledged from the DFG under grant Se 1008/6 and from the NanoScale initiative of the FU Berlin. Supercomputer time was provided by the Julich supercomputing center under grant 4781. Molecular graphics images were produced using VMD. ${ }^{44}$ Numerical diagonalization of the spin Hamiltonian was performed using the Eigen C++ template library ${ }^{45}$ and parallelized using Open MPI. ${ }^{46}$

\section{References}

(1) Pavone, M.; Cimino, P.; De Angelis, F.; Barone, V. J. Am. Chem. Soc. 2006, 128, 4338- 
47.

(2) Pavone, M.; Cimino, P.; Crescenzi, O.; Sillanpää, A.; Barone, V. J. Phys. Chem. B 2007, 111, 8928-39.

(3) Al Derzi, A. R.; Fau, S.; Bartlett, R. J. J. Phys. Chem. A 2003, 107, 6656-6667.

(4) Barone, V.; Brustolon, M.; Cimino, P.; Polimeno, A.; Zerbetto, M.; Zoleo, A. J. Am. Chem. Soc. 2006, 128, 15865-73.

(5) Lund, A.; Macomber, L. D.; Danilczuk, M.; Stevens, J. E.; Schlick, S. J. Phys. Chem. B 2007, 111, 9484-91.

(6) Barone, V.; Cimino, P. J. Chem. Theory Comput. 2009, 5, 192-199.

(7) Gauss, J.; Kállay, M.; Neese, F. J. Phys. Chem. A 2009, 113, 11541-9.

(8) Arbuznikov, A. V.; Kaupp, M.; Malkin, V. G.; Reviakine, R.; Malkina, O. L. Phys. Chem. Chem. Phys. 2002, 4, 5467-5474.

(9) Neese, F. J. chem. Phys. 2001, 115, 11080.

(10) Pauwels, E.; Asher, J.; Kaupp, M.; Waroquier, M. Phys. Chem. Chem. Phys. 2011, 13, 18638-46.

(11) Heller, J.; Elgabarty, H.; Zhuang, B.; Sebastiani, D.; Hinderberger, D. J. Phys. Chem. B 2010, 114, 7429-38.

(12) Improta, R.; Barone, V. Chem. Rev. 2004, 104, 1231-54.

(13) Nitzan, A. Chemical Dynamics in Condensed Phases: Relaxation, Transfer, and Reactions in Condensed Molecular Systems; Oxford University Press, 2006.

(14) Simons, J.; Nichols, J. Quantum Mechanics in Chemistry; Oxford University Press, USA, 1997.

(15) Poole, C. P.; Farach, H. A. Relaxation in magnetic resonance: dielectric and mössbauer applications; Academic Press, 1971.

(16) Stoneham, A. M. Rev. Mod. Phys. 1969, 41, 82108.

(17) Asher, J. R.; Doltsinis, N. L.; Kaupp, M. J. Am. Chem. Soc. 2004, 126, 9854-61.
(18) Asher, J. R.; Kaupp, M. ChemPhysChem 2007, 8, 69-79.

(19) Dixit, S.; Crain, J.; Poon, W. C. K.; Finney, J. L.; Soper, a. K. Nature 2002, 416, 829-32.

(20) Pilbrow, J. R. Appl. Magn. Reson. 1994, 6, 161181.

(21) VandeVondele, J.; Krack, M.; Mohamed, F.; Parrinello, M.; Chassaing, T.; Hutter, J. Comput. Phys. Commun. 2005, 167, 103-128.

(22) VandeVondele, J.; Hutter, J. Chem. Phys. 2007, 127, 114105.

(23) Perdew, J.; Burke, K.; Ernzerhof, M. Phys. Rev. Lett. 1996, 77, 3865-3868.

(24) Grimme, S. J. Comput. Chem. 2006, 27, 1787-99.

(25) Bussi, G.; Donadio, D.; Parrinello, M. Chem. Phys. 2007, 126, 014101.

(26) Kutzelnigg, W.; Fleischer, U.; Schindler, M. In Deutereum and Shift Calculation; Diehl, P., Fluck, E., Günther, H., Kosfeld, R., Seelig, J., Eds.; NMR Basic Principles and Progress; Springer: Heidelberg, 1990; Vol. 23; pp 165-262.

(27) Lippert, G.; Hutter, J.; Parrinello, M. Theor. Chem. Acc. 1999, 103, 124-140.

(28) Weber, V.; Iannuzzi, M.; Giani, S.; Hutter, J.; Declerck, R.; Waroquier, M. Chem. Phys. 2009, 131, 014106 .

(29) Genovese, L.; Deutsch, T.; Neelov, A.; Goedecker, S.; Beylkin, G. Chem. Phys. 2006, $125,074105$.

(30) Neese, F. Chem. Phys. 2005, 122, 34107.

(31) Barone, V. In Recent Advances In Density Functional Methods; Chong, D. P., Ed.; World Scientific Publishing Co. Pte. Ltd., 1995; Vol. 1; Chapter 8, pp 287-334.

(32) R Core Team, R: A Language and Environment for Statistical Computing. R Foundation for Statistical Computing: Vienna, Austria, 2012; ISBN 3-900051-07-0.

(33) using Fortran code by Alan Miller, T. L. leaps: regression subset selection. 2009; R package version 2.9. 
(34) Venables, W. N.; Ripley, B. D. Modern Applied Statistics with $S$, 4th ed.; Springer: New York, 2002; ISBN 0-387-95457-0.

(35) Frisch, M. J. et al. Gaussian 09 Revision A.02. Gaussian Inc. Wallingford CT 2009.

(36) Stoll, S.; Schweiger, A. Chem. Phys. Lett. 2003, 380, 464-470.

(37) Lebedev, V. I.; Laikov, D. Dokl. Math.; 1999; Vol. 59; pp 477-481.

(38) Stevensson, B.; Edén, M. J. Magn. Reson. 2006, $181,162-76$.

(39) Edén, M. Concept. Magn. Reson. 2003, 18A, 2455.

(40) Hinderberger, D.; Spiess, H. W.; Jeschke, G. J. Phys. Chem. B 2004, 108, 3698-3704.

(41) Declerck, R.; Van Speybroeck, V.; Waroquier, M. Phys. Rev. B 2006, 73, 1-8.

(42) Malkina, O. L.; Vaara, J.; Schimmelpfennig, B.; Munzarova, M.; Malkin, V. G.; Kaupp, M. J. Am. Chem. Soc. 2000, 122, 9206-9218.

(43) Sinnecker, S.; Rajendran, A.; Klamt, A.; Diedenhofen, M.; Neese, F. J. Phys. Chem. A 2006, 110, 2235-45.

(44) Humphrey, W.; Dalke, A.; Schulten, K. J. Mol. Graphics 1996, 14, 33-38.

(45) Guennebaud, G.; Jacob, B. Eigen v3; 2012.

(46) Gabriel, E.; Fagg, G. E.; Bosilca, G.; Angskun, T.; Dongarra, J. J.; Squyres, J. M.; Sahay, V.; Kambadur, P.; Barrett, B.; Lumsdaine, A.; Castain, R. H.; Daniel, D. J.; Graham, R. L.; Woodall, T. S. Proceedings, 11th European PVM/MPI Users' Group Meeting; 2004; pp 97104. 